\title{
Checking Formal Specifications by Testing
}

\author{
Dick Hamlet* \\ Fulbright Scholar \\ Department of Mathematics \\ National University of Ireland, Galway \\ Invited talk, July 2, 1999
}

\begin{abstract}
Formal specification methods hold promise for bridging the wide gap between an intuitive idea for solving a problem by computer, and the executable program that attempts to do the job. The use of formalism is itself a good thing, allowing professionals to understand and analyze their work better. However, formal methods are an aid to human effort, not a panacea. Conventional software testing can be an ideal complement to formally directed development. Tests are concrete and immediately comprehensible to end users, and they are unlikely to miss mistakes because of a pernicious correlation with the formal work.

Research is needed on ways to make formal specifications and testing work together to realize the potential of both. Tests should serve to increase confidence that a formal method has been correctly applied. Such tests would free the developers from tedious checking of formalism details, and the success of only a few tests would have real significance for the software's correctness.

As an example of a formalism/testing partnership, this talk describes joint work with Sergio Antoy [4] on automatically checking a conventional implementation of an abstract data type against its formal algebraic specification.
\end{abstract}

\section{Formal Methods and Testing}

In the "old days" when formal methods were identified with proving an implementation program correct with respect to a first-order logic specification, this proving was thought to be antithetical to testing. The two methods were viewed as being at opposite ends of a spectrum: the formal work was abstract, precise, and concerned with the function the program computes; testing was practical, sloppy, and concerned only with finite support for that function.

A more modern viewpoint considers the two subjects complementary. Today's formal methods are still abstract and precise, but now much more broadly concerned with expressing and reasoning about specifications. Testing today is still practical and sometimes sloppy, but it can be the most technical and precise of the other software-development arts. Specification and testing remain at

\footnotetext{
${ }^{*}$ On leave from Portland State University, Department of Computer Science and Center for Software Quality Research, Portland, OR 97207, USA
} 
opposite ends of the development time-line, so there is maximum scope for testing to serve as a check on the whole process.

To mention a promising interaction in which testing benefits from a formal specification, consider automatic random testing. This idea is to subject code to inputs chosen at random, where the random generation of test points is planned and automated using a formal description of a program's input domain. To be effective, random testing must employ far more test points than the usual "by hand" tests. Hence it is practical only if the results can be automatically checked, and here again a formal specification is the answer. Many formal specifications are effective in that they provide a decision procedure for whether or not any input-output pair conforms to the specification.

In the other direction, testing has the potential to detect mistakes in software development, mistakes which may arise from poor communication between end users and software experts (that is, the formal specifications are for the wrong problem), and mistakes in carrying through development (that is, the formal specifications have not been properly followed). Here the technical nature of both disciplines comes to the fore: it should be possible to devise tests that pinpoint common mistakes, and if the tests succeed, to gain great confidence that nothing is wrong.

\subsection{Formal Methods}

Formal methods (hereafter "FMs") are broadly any mathematical techniques used in program development. The intent is clearly to bring abstract thought to bear on the difficult problems of describing what a program is supposed to do and seeing that it does just that. The common narrower meaning of FMs is mathematics for stating and reasoning about specifications. Some FMs have a constructive, algorithmic character, while others are more abstract. At the abstract extreme, intuitive set theory provides most of the mathematical abstractions needed for describing program meaning. At the other extreme, a finite-state-machine description allows the mechanical generation or checking of many aspects of specification. The $\mathrm{Z}$ language is close to the abstract end, while the SCR method is near the constructive end. In between, methods may be designed for facilitating mechanical proofs, but not necessarily in a decidable theory; PVS is one such FM.

To usefully interact with software testing, a FM must be capable of serving as an oracle. An oracle is a means of attempting to decide of any pair $(x, y)$ whether or not on input $x, y$ is a result consistent with the specification. An effective oracle is a mechanical decision procedure.

\section{$1.2 \quad$ Testing}

In principle, a test is a finite set of inputs for a program. A test succeeds if the program outputs are consistent with the specification. A failure is a test point on which the program output is inconsistent with the specification.

Since testing deals only with a finite input set, it cannot establish general properties of the program being tested. But in random testing it is possible to obtain statistical results such as a bound on the probability of failure. A clever choice of test points can also be used to probe for likely failures. In fault-based testing, test points are chosen so as to unambiguously answer worrisome questions about program behavior. For example, a programmer may be concerned about the conditional expression terminating a loop: should the comparison be " $\leq$ " or " $<$ "? It is usually possible to find a single test case that will succeed only if the conditional is correct.

\subsection{Research Agenda}

FMs and testing each has difficult technical problems that define current research. It may be that each area can help the other. 


\subsubsection{FMs in aid of testing}

By far the most important interaction between FMs and testing is that a FM provides an oracle. The testing literature almost universally assumes that an oracle is available for the program being tested, but in practice this assumption is mostly false.

"Specification-based" tests are recommended as the only ones capable of detecting programming errors of omission, and as reflecting what a program is expected to accomplish. Not the least important property of specification-based tests is that they can be devised early in the development process, and in parallel with the rest of development. Even the most non-constructive FMs have a well defined syntax that allows their specifications to be printed in a standard format, and to be processed mechanically; the syntax is often enough to permit automatic test generation.

\subsubsection{Testing in aid of FM}

FMs have a number of difficulties in principle, and testing can be of some help for each:

Wrong specification. By far the strongest argument against the use of FMs is that they, like any specification method, may fail to capture the intuitive requirements of the software end user. These user requirements are intrinsically vague and imprecise, and in sharpening them as any FM must, human beings may get it wrong. Testing can help by generating revealing, representative cases, which unsophisticated users can understand, to try out the formal specification.

Improper use of the FM. Every FM has pitfalls that allow its practitioners to create apparently meaningful specifications that contain technical mistakes. For example, in any "declarative" FM, it will be possible to write a specification that is correctly implemented by almost any program; at the other extreme a specification may have no correct implementations. As experience with a FM is gained, one might hope to make a catalog of "common blunders," and to find corresponding tests, by analogy to "fault-based" tests for programs, that necessarily expose them.

Inconsistent implementation. Tests are conducted on the final product of software development: the program. They therefore check how faithfully the design and code have followed the specification. The example presented in section 2 to follow falls in this category.

\section{Example: Self-checking ADT Code}

As an example of the potential benefits of interaction between the FMs and testing communities, this section summarizes research work primarily done by my colleague Sergio Antoy, to appear elsewhere [4]. In that research, an abstract data type (ADT) is specified using a formal algebraic specification, the ADT is implemented by hand in a conventional programming language like $\mathrm{C}^{++}$, and a largely automatic scheme is used to instrument the program so that as it executes, it checks each result against the specification.

The exposition of Antoy's scheme uses the example of a finite set of integers, as taken from Stroustrup's $\mathrm{C}++$ textbook. This ADT has operations given by the signature in figure 1 .

\subsection{Correctness of an ADT}

An implementation of an ADT is correct iff certain diagrams for its operations commute. These diagrams display the "abstract" world of the ADT specification, and the "concrete" world of pro- 


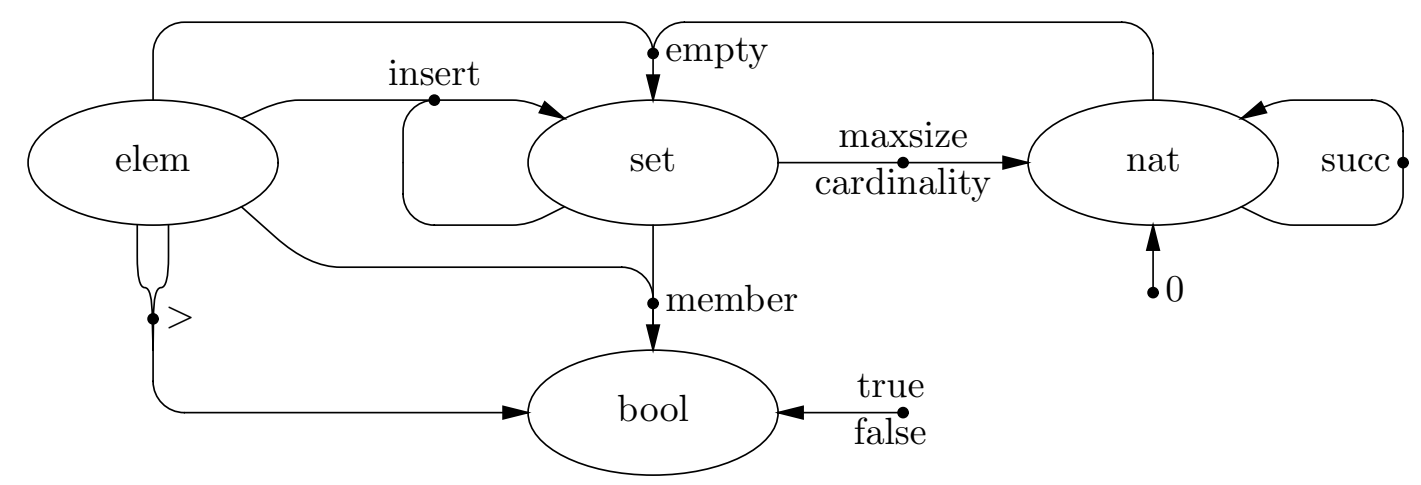

Figure 1: Signature of a generic, bounded ADT set.

gram states in a computer. The two worlds are connected by a so-called "representation" mapping. If a representation mapping exists such that the diagram commutes, it establishes the correctness of the implementation. For example, the diagram for the "membership" operation on sets and their elements is shown in figure 2. It commutes iff starting at the lower left with any element $x$ and any

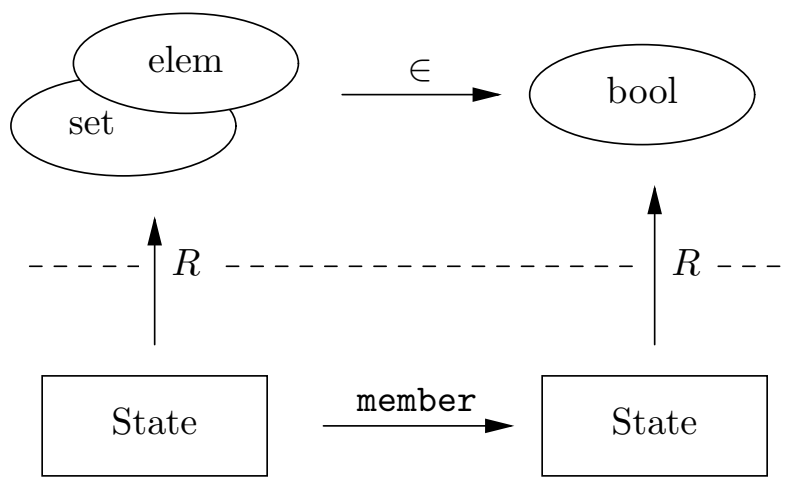

Figure 2: Commuting diagram for the member operation of the ADT set.

set $S$ (values in the state), and passing around the diagram in the two possible ways to the upper right, the results agree:

$$
R(x) \in R(S) \Longleftrightarrow R(b),
$$

where $b$ is the value in the state at the lower right that results from calling member with argument values $x$ and $S$.

It was Antoy's idea that it would be possible to automate checking of the commuting diagram for particular values of $x$ and $S$ if the representation mapping were made explicit, that is, if it were programmed as part of the $\mathrm{C}^{++}$implementation of the $\mathrm{ADT}$. In addition to $R$, checking the diagram requires the ability to compute:

The abstract function. ( $\in$ in figure 2.) The ADT is specified by a set of equational axioms. These axioms describe a ground word algebra in the names of the operations and conventional representations of the integers, and allow words to be reduced to normal form, using the axioms as left-to-right rewriting rules. (Antoy uses a restricted axiom form that guarantees that the rewrite theory will be identical to the equational theory.) Hence the abstract function at the top of the diagram can be computed by rewriting (in the word algebra). 
The concrete function. (member in figure 2.) The $\mathrm{C}^{++}$implementation provides the ability to compute all concrete functions.

\subsection{Equational Specification}

To give the flavor of the formal specification method, here is a part (about half) of the specification for the set of integers:

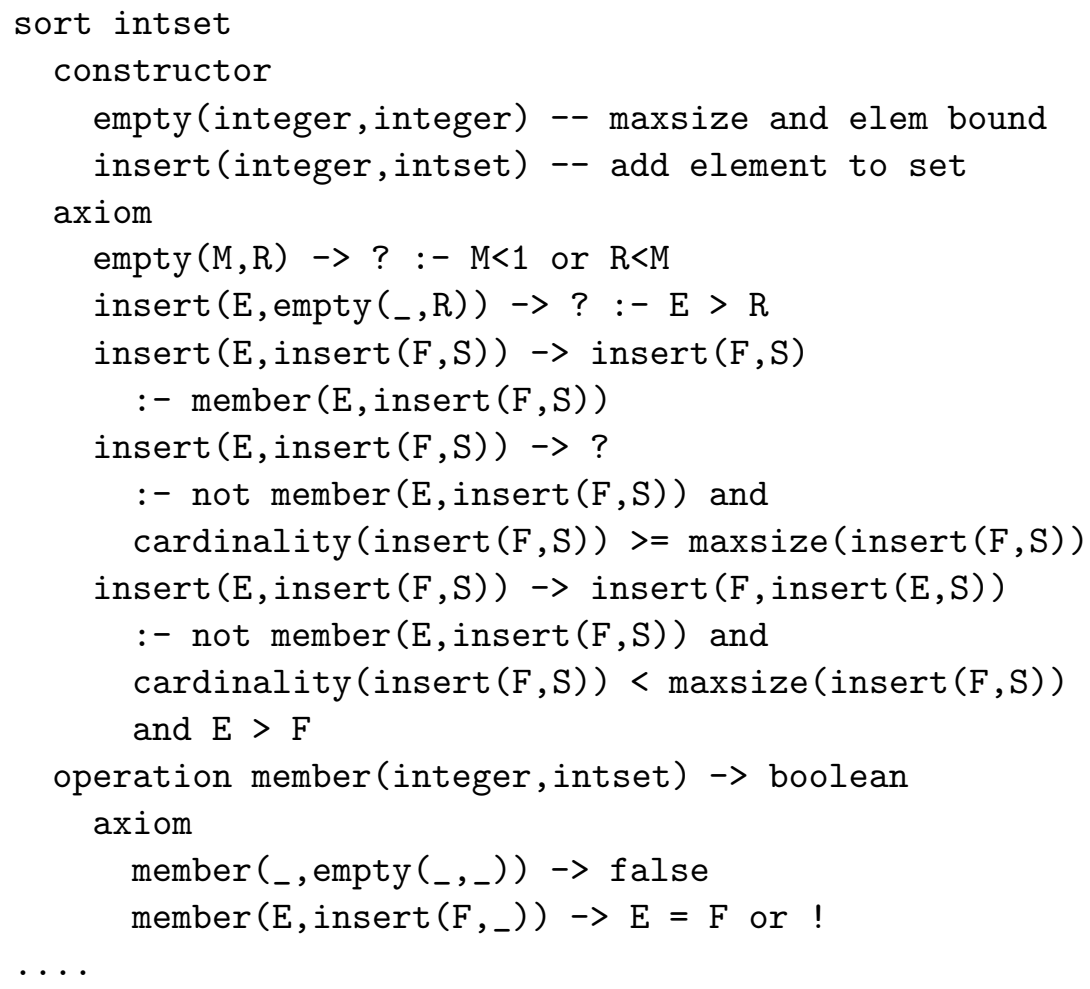

Notations: The "?" notation signals an exception that terminates rewriting. Rules may have a guard following ": -" to select that rule.

The fourth rule for insert, for example, applies in the case that the inserted element is not already in the set, the set is not too large, and the inserted element is larger than the one currently at the beginning of the word being rewritten. Thus this rule specifies that the canonical form will have the set elements in ascending order.

\subsection{The "Direct" (Rewriting) Implementation}

The rules of the specification can be used to compute normal forms in the word algebra. This computation is the same for all specifications, except for the particular rules to be used. A possible implementation strategy for the rewriting is to store the algebraic words in a tree structure, and rewrite by manipulating the tree. We call this the direct implementation because it comes directly from the specification, and it can be mechanically compiled into $\mathrm{C}^{++}$code.

For example, here is how a portion of the direct implementation might appear for the set example:

typedef int elem; // kind of generic

enum tag \{ EMPTY, INSERT \}; // discriminant tokens 


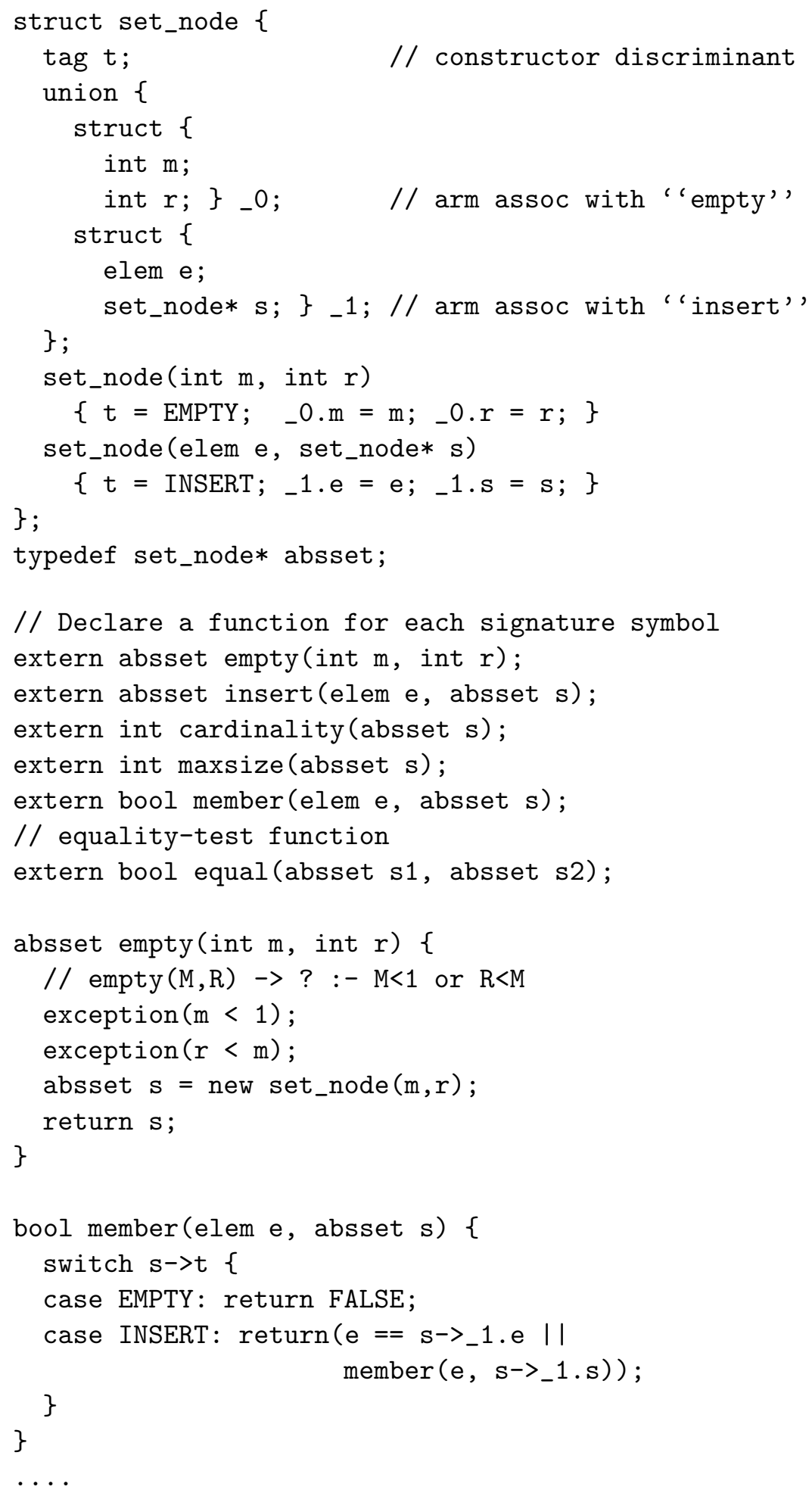

\subsection{Additions to the Hand Implementation}

Finally, to complete the code package for a self-checking ADT, the implementation code that has been written by hand must be instrumented with some small changes to connect its computations to 
those of the direct implementation. These (1) add code for the explicit representation function, (2) call the representation function to convert state values to the abstract world, and (3) compare the results of the direct-implementation computation with this converted result. With this additional code in place, the by-hand implementation will check each of its computations and report any disagreement with the specification. The additional code required for the set example is indicated below.

\section{Representation function}

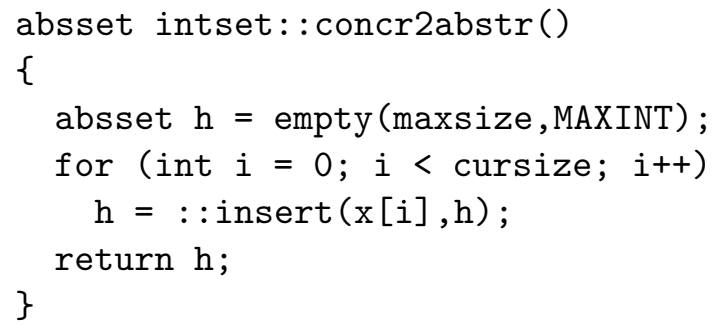

\section{Verification code in by-hand implementation}

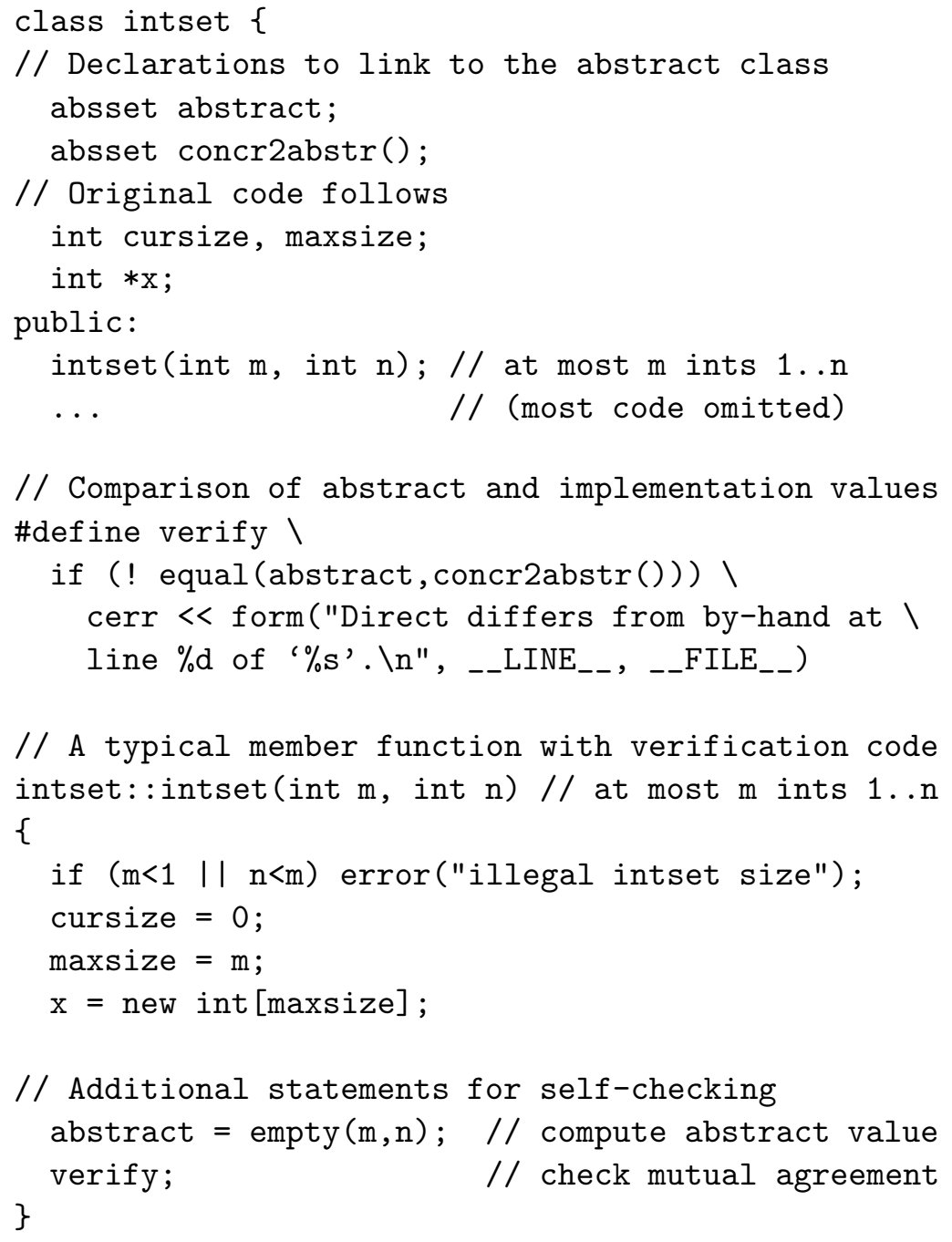




\subsection{Summary of the Self-checking Code}

Figure 3 summarizes the form taken by a complete self-checking implementation, using the set example.

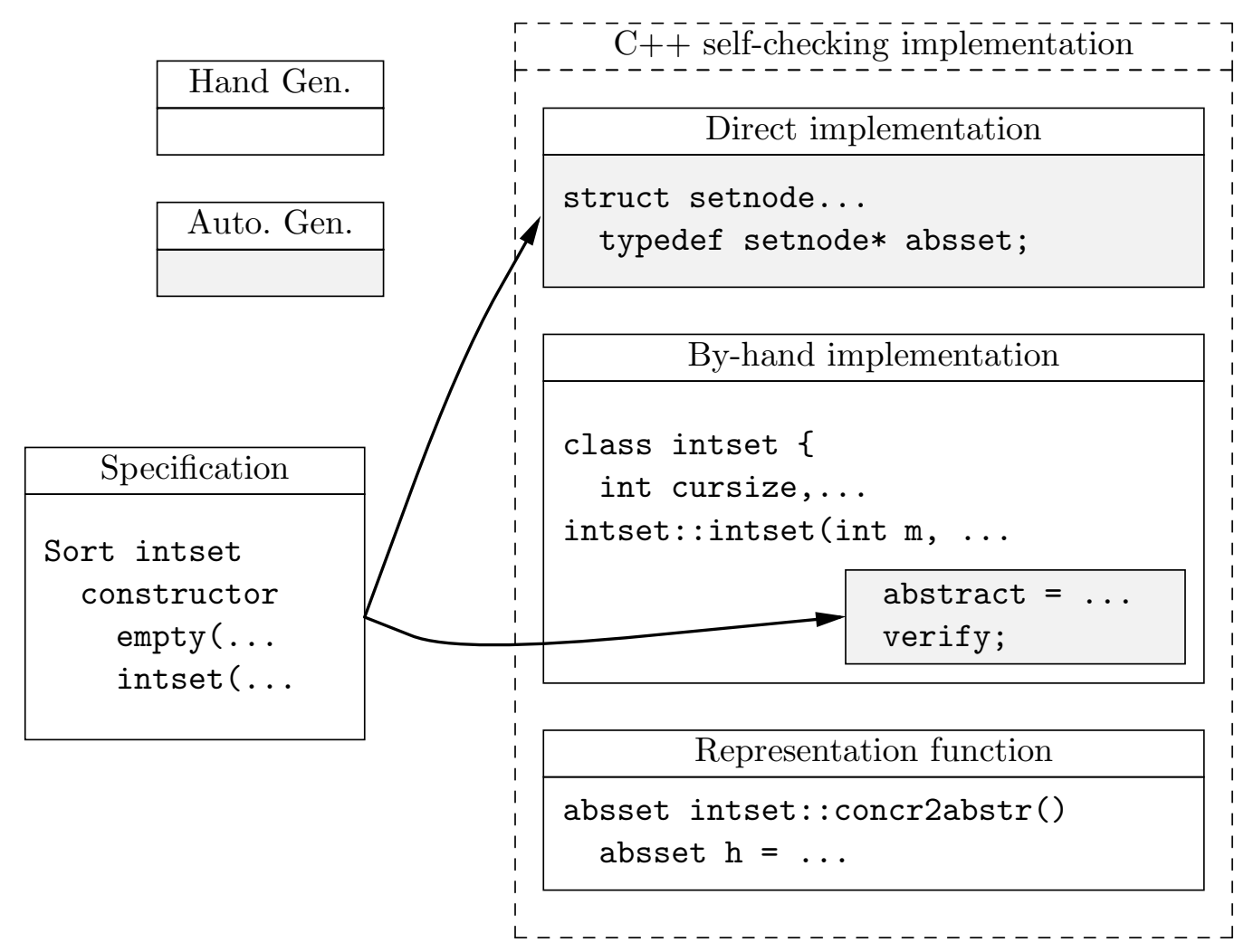

Figure 3: Construction of the self-checking implementation.

\subsection{Discussion of Self-checking}

The self-checking scheme we have described connects a formal specification and a conventional implementation to provide for automatic run-time checking of each computed result. Thus the hand implementation is provided with an effective test oracle, which allows automated testing. A simple driver program that selects random inputs can be used to conduct far more tests than are usually performed on any code. In experiments with a part of the Java library, we were able to execute $10^{6}$ tests in about 20 minutes on a modern laptop.

Self-checking also provides a kind of ultimate multi-version programming. There is no apparent connection between the data structures, algorithms, or other aspects of the way in which the two implementations, the direct using rewriting, and the by-hand using conventional techniques, produce their results. Thus if they agree, it provides some assurance that neither of them is doing the wrong thing as judged by intuition.

It is more controversial to recommend that self-checking code be carried from the test phase of development into released code. The conventional wisdom is that all checks should be removed from "production" code in the interests of speed. (Perhaps the embarrassment of a program that announces that it has just made a mistake is another factor in removing checks.) Rewriting in the direct implementation is sometimes much slower than a conventional implementation, but it is well 
known that only a very small part of any program lies on the path of time-critical execution, and it would be easy enough to omit checking only on this path. What to do when the implementation disagrees with the specification is a more interesting question.

First, when a failure is detected in a running production program, information can be logged so that fixing the program later is easier. But what can be done about the failed calculation, on which real-time decisions may be riding, and ultimately even human lives? Our position is that it is far better to know that a result is wrong than to erroneously believe that it is right. One possibility is simply to try again. It may be that the failure is isolated, and slight differences in sensor readings or timing may produce a correct result. In the case of a hard failure, there may be a human in the loop who can be given control. But absent all such plausible outcomes, we believe that the potential for failure puts a necessary pressure on the software designer. If the system architect knows that an ADT might fail, then the system design must try to take failure into account, and consider retries, calling for human help, etc. The resulting code will be much safer than if the ADT were blindly trusted. Ultimately, if the architect sees a case in which failure will necessarily lead to disaster, it will be necessary to use extreme measures, for example to rigorously prove that this case cannot occur, or even to inform the customer that perhaps the computer application should be reconsidered.

\section{Conclusion}

Formal specification methods and software testing have the potential to contribute much to each other. As the two most objective, technical aspects of software development, they are natural allies.

This paper has indicated an example of the way in which a formal algebraic specification can enable self-checking of conventional code. This application is actually the least interesting of the possibilities for interaction between the disciplines. The greatest potential seems to lie in finding "fault-based" tests that will convince a developer that a given FM has been properly applied, and that its potential pitfalls have been avoided.

The example has only been sketched here; the published paper [4] should be consulted for details. The bibliography to follow has been condensed from that paper.

\section{References}

[1] S. Antoy. Design strategies for rewrite rules. In $C T R S^{\prime} 90$, Montreal, Canada, June 10-14 1990. Springer-Verlag.

[2] S. Antoy, P. Forcheri, and M.T. Molfino. Specification-based code generation. In 23rd Hawaii Int'l Conf. on System Sciences, pages 165-173, Kailua-Kona, Hawaii, Jan. 3-5 1990.

[3] S. Antoy and J. Gannon. Using term rewriting systems to verify software. IEEE Trans. Soft. Eng., 20(4):259-274, 1994.

[4] Sergio Antoy and Dick Hamlet. Automatically checking an implementation against its formal specification. IEEE Trans. on Soft. Eng., to appear, 1999.

[5] J. A. Bergstra, J. Heering, and P. Klint. Algebraic Specification. Addison-Wesley, Wokingham, England, 1989.

[6] M. Bidoit, H.-J. Kreowski, P. Lescanne, F. Orejas, and D. Sannella, editors. Algebraic System Specification and Development. Springer-verlag, 1991. Lect. Note in Comp. Sci., Vol. 501. 
[7] N. Choquet. Test data generation using a Prolog with constraints. In Workshop on Software Testing, pages 132-141, Banff, Canada, 1986.

[8] M. D. Davis and E. J. Weyuker. A formal notion of program-based test data adequacy. Information and Control, 56:52-71, 1983.

[9] N. Dershowitz. Termination. In RTA'85, pages 180-223, Dijon, France, May 1985. SpringerVerlag.

[10] N. Dershowitz, M. Okada, and G. Sivakumar. Confluence of conditional term rewrite systems. In $C T R S^{\prime} 87$, pages 31-44, Orsay, France, 1987. Springer-Verlag.

[11] R-K. Doong and P. Frankl. Case studies on testing object-oriented programs. In Proceedings of the Symposium on Testing, Analysis, and Verification (TAV4), pages 165-177, Victoria, B.C., 1991.

[12] M. Ehrig and B. Mahr. Fundamentals of Algebraic Specification 1. Springer-Verlag, Berlin, 1985.

[13] J. Gannon, R. Hamlet, and P. McMullin. Data abstraction implementation, specification, and testing. ACM Trans. Prog. Lang. and Systems, 3:211-223, 1981.

[14] J. D. Gannon, R. G. Hamlet, and H. D. Mills. Theory of modules. IEEE Trans. on Soft. Eng., 13, 1987.

[15] M.-C. Gaudel and B. Marre. Generation of test data from algebraic specifications. In Second Workshop on Software Testing, Verification, and Analysis, pages 138-139, Banff, Canada, 1988.

[16] S. Gerhart. Test generation method using prolog. Technical Report TR 85-02, Wang Institute of Graduate Studies, 1985.

[17] J. Goguen, J.-P. Jouannaud, and J. Meseguer. Operational semantics of order-sorted algebras. In W. Brauer, editor, CALP'85. Springer-Verlag, 1985.

[18] J. A. Goguen and T. Winkler. Introducing OBJ3. Technical Report SRI-CSL-88-9, SRI International, Menlo Park, CA, 1988.

[19] J.A. Goguen, J.W. Thatcher, and E.G. Wagner. An initial algebra approach to the specification, correctness, and implementation of abstract data types. In R.T. Yeh, editor, Current Trends in Programming Methodology, volume 4, pages 80-149. Prentice Hall, Englewood Cliff, NJ, 1978.

[20] J. V. Guttag. Notes on type abstraction. IEEE Trans. on Soft. Engineering, 6:13-23, 1980.

[21] J. V. Guttag and J. J. Horning. The algebraic specification of abstract data types. Acta Informatica, 10:27-52, 1978.

[22] J. V. Guttag, E. Horowitz, and D. Musser. The design of data type specifications. In R. T. Yeh, editor, Current Trends in Programming Methodology, volume 4, pages 60-79. Prentice-Hall, Englewood Cliff, NJ, 1978.

[23] J.V. Guttag, E. Horowitz, and D. Musser. Abstract data types and software validation. CACM, 21:1048-1064, 1978. 
[24] D. Hamlet. Random testing. In J. Marciniak, editor, Encyclopedia of Software Engineering, pages 970-978. Wiley, New York, 1994.

[25] C. L. Heitmeyer, R.D. Jeffords, and B. G. Labaw. Automated consistency checking of requirements specifications. ACM Trans. on Software Eng. and Methodology, 5:231-261, 1996.

[26] C. A. R. Hoare. Proof of correctness of data representations. Acta Informatica, 1:271-281, 1972 .

[27] D. Hoffman. Hardware testing and software ICs. In Pacific Northwest Software Quality Conference, pages 234-244, Portland, OR, 1989.

[28] D. Hoffman and C. Brealey. Module test case generation. In Third Symposium on Software Testing, Analysis, and Verification, pages 97-102, Key West, FL, 1989.

[29] G. Huet. Confluent reductions: Abstract properties and applications to term-rewriting systems. JACM, 27:797-821, 1980.

[30] Gérard Huet and Jean-Marie Hullot. Proofs by induction in equational theories with constructors. JCSS, 25:239-266, 1982.

[31] J. W. Klop. Term rewriting systems. Technical Report CS-R9073, Stichting Mathematisch Centrum, Amsterdam, The Netherlands, 1990.

[32] J.W. Klop. Term rewriting systems: a tutorial. Bulletin of the EATCS, 32:143-182, 1987.

[33] J. C. Knight and N. G. Leveson. An experimental evaluation of the assumption of independence in multi-version programming. IEEE Trans. on Soft. Eng., 12:96-109, 1986.

[34] D.E. Knuth and P.B. Bendix. Simple word problems in universal algebras. In J. Leech, editor, Computational Problems in Abstract Algebras, pages 263-297. Pergamon Press, New York, NY, 1970.

[35] N. G. Leveson, S. S. Cha, J. C. Knight, and T. J. Shimeall. The use of self checks and voting in software detection: An empirical study. IEEE Trans. on Soft. Eng., 16:432-443, 1990.

[36] M. J. O’Donnell. Equational Logic as a Programming Language. MIT Press, 1985.

[37] S. Sankar. Run-time consistency checking of algebraic specifications. In Proceedings of the Symposium on Testing, Analysis, and Verification (TAV4), pages 123-129, Victoria, B.C., 1991.

[38] B. Stroustrup. The $C^{++}$Programming Language. Addison-Wesley, Reading, MA, 1986.

[39] C. Wang and D. R. Musser. Dynamic verification of $\mathrm{C}^{++}$generic algorithms. IEEE Trans. Soft. Eng., 23(5):314-323, 1997. 